\title{
AVALIAÇÃO DA EFICIÊNCIA DA RADIAÇÃO ULTRAVIOLETA NA ESTERILIZAÇÃO DE EMBALAGENS PLÁSTICAS
}

\author{
Evaluation of ultraviolet radiation in the sterilization of plastic packaging
}

\author{
Fernanda Antunes Alexandre', José de Assis Fonseca Faria², Claudio Fernandes Cardoso ${ }^{3}$
}

\begin{abstract}
RESUMO
O uso da radiação ultravioleta é um dos vários processos físicos que podem ser utilizados para a esterilização ou na higienização de superfícies que irão entrar em contato com alimentos. Objetivou-se, nesta pesquisa a avaliação da eficiência da radiação ultravioleta (UV) na esterilização de superfícies de embalagens para alimentos visando a redução da contaminação microbiológica. Tampas e filmes plásticos foram irradiados em uma fonte de ultravioleta emitindo comprimentos de ondas a $254 \mathrm{~nm}$, no interior de uma câmara especialmente projetada para este estudo, através de um delineamento fatorial para testar as variáveis: tempo de exposição e intensidade de irradiação (distância da fonte). Avaliou-se o número de reduções decimais de esporos de Bacillus subtilis inoculados na superfície das embalagens. As reduções decimais obtidas não foram altas, variaram de 0,21 a 2,47, mas o uso da lâmpada UV foi altamente eficiente para tempo de exposição superior a $30 \mathrm{~s}$ e distância mínima de $20 \mathrm{~cm}$ da fonte, causando a redução total da carga microbiana inoculada.
\end{abstract}

Termos para indexação: Radiação UV, esterilização, embalagens plásticas.

\section{ABSTRACT}

Ultraviolet radiation is one of the major physical processes used to sterilize or to clean surfaces that will be in contact of foods. This research evaluated the efficiency of ultraviolet (UV) radiation to sterilize surfaces of plastic packaging materials based on the reduction of microbial counts. Plastic closures and films were irradiated by exposing them to lamps emitting UV radiation at 254 $\mathrm{nm}$ in an apparatus specially constructed for this study. Using an experimental design in order to investigate the effect of exposure time and distance from the source, the reduction number of Bacillus subtilis spores inoculated to the package surfaces were evaluated. The UV radiation efficiency was not high for short exposition time, presenting reduction number ranged from 0.21 to 2.47 , but it was very efficient when exposed to more than $30 \mathrm{~s}$ at a distance of $20 \mathrm{~cm}$.

Index terms: UV radiation, sterilization, plastic packaging.

(Recebido em 23 de outubro de 2006 e aprovado em 27 de maio de 2008)

\section{INTRODUÇÃO}

As inovações tecnológicas no setor de alimentos têm evoluído bastante nas últimas décadas e, dentre elas, destacam-se os sistemas de processamento e acondicionamento assépticos. Em geral, os sistemas assépticos envolvem as etapas de esterilização do material de embalagem; esterilização comercial do produto; acondicionamento do produto assepticamente; fechamento e manutenção da integridade da embalagem (FARIA, 1993).

Certas embalagens podem apresentar-se estéreis logo após sua fabricação, como as garrafas e tampas plásticas devido às altas temperaturas alcançadas no processo de extrusão termoplástica, mas podem ser facilmente recontaminadas durante o manuseio e estocagem, por causa da deposição de partículas e microrganismos do ar. Em geral, os níveis de contaminação variam entre 1-10 UFC/ $\mathrm{cm}^{2}$ de superfície do material que não tenha sido manuseado, apresentando alta contagem de bactérias gram negativas (JOYCE, 1993).

Basicamente, a esterilização da superfície interna da embalagem, que entrará em contato com o alimento no acondicionamento asséptico, é obtida pela ação de agentes químicos e físicos ou pela combinação deles. Pesquisas têm comprovado que o grau de esterilização obtido depende do nível de contaminação inicial, área da embalagem e eficiência do processo de esterilização (ABREU \& FARIA, 2004; FARIA, 2001).

Denomina-se irradiação ao processo de aplicação de energia radiante a um alvo qualquer, como por exemplo, um alimento ou uma embalagem (INGRAM \& ROBERTS, 1980). Tal tratamento tem sido utilizado em

\footnotetext{
Engenheira de Alimentos - Departamento de Tecnologia de Alimentos/DTA - Faculdade de Engenharia de Alimentos - Universidade Estadual de Campinas/UNICAMP - Cx. P. 6121 - 13083-970 - Campinas, SP - fernandaantunes@hotmail.com

${ }^{2}$ Engenheiro Agrônomo, Doutor em Ciência dos Alimentos, Professor Titular - Departamento de Tecnologia de Alimentos/DTA - Faculdade de Engenharia de Alimentos - Universidade Estadual de Campinas/UNICAMP - Cx. P. 6121 - 13083-970 - Campinas, SP - assis@fea.unicamp.br ${ }^{3}$ Engenheiro de Alimentos, Mestre - Departamento de Tecnologia de Alimentos/DTA - Faculdade de Engenharia de Alimentos - Universidade Estadual de Campinas/UNICAMP - Cx. P. 6121 - 13083-970 - Campinas, SP - cardocos@gmail.com
} 
processos assépticos, tanto para a esterilização do ar quanto para a esterilização do material da embalagem (WILLHOFT, 1993).

O tratamento por radiação ultravioleta tem um longo e eficiente histórico no controle microbiológico do ar. Em aplicações como tratamento de bebidas e superfícies ele tem se destacado, embora para superfícies de alimentos o seu uso vise a extensão da vida útil do produto (KONINKLIJKE PHILIPS ELETRONICS, 2007).

A irradiação ultravioleta tem efeito microbiocida se for utilizada com intensidade e tempo de exposição suficiente, encontrando aplicações diversas como na esterilização do ar, superfícies de equipamentos e em embalagens de alimentos. Fontes com comprimentos de ondas inferiores a $200 \mathrm{~nm}$ são ineficientes, visto que as ondas são rapidamente absorvidas pelo oxigênio e pela água. As irradiações ultravioletas na faixa de 210 e $330 \mathrm{~nm}$ são mais eficientes como germicidas por serem absorvidas pelas proteínas e ácidos nucléicos, provocando o rompimento de cromossomo, mutações genéticas e inativação de enzimas e, conseqüentemente, a morte da célula (CARDOSO, 2007).

Em geral, a radiação ultravioleta tem se mostrado como a forma mais rápida, confiável, efetiva, econômica e ambientalmente segura no tratamento de superfícies e líquidos. Além disso, o uso combinado da radiação ultravioleta e dos desinfetantes químicos têm apresentado um efeito sinérgico no controle de microrganismos, especialmente em casos onde o residual do agente químico deve ser controlado em taxas mínimas ou estar ausente (ABREU \& FARIA, 2004; SIEMENS WATER TECHNOLOGIES, 2007).

O método mais prático de gerar radiação UV é pela passagem de descargas elétricas através de vapor de mercúrio a baixa pressão dentro de tubos de vidro especiais, conhecidos comercialmente como lâmpadas germicidas. Segundo ICMSF (1980), uma lâmpada germicida de $50 \mathrm{~W}$, posicionada a $1 \mathrm{~m}$ de distância de um alvo, tem uma intensidade de $100 \mathrm{~mW} / \mathrm{cm}^{2}$. As doses de irradiação absorvidas são medidas em energia, expressas em ergs ou $\mathrm{mW} . \mathrm{s} / \mathrm{cm}^{2}$

O sucesso da esterilização de embalagens pela radiação UV depende de uma série de fatores, sendo que a superfície a ser esterilizada deve estar extremamente limpa. Como os raios UV têm um poder pequeno de penetração, podem ser facilmente absorvidos por partículas sólidas na superfície da embalagem. Sendo assim, os microrganismos podem ser protegidos por poeira, provocando o chamado "efeito sombra" (REUTER, 1993). Além disso, tanto a distância como o ângulo de incidência da fonte à superfície a ser tratada devem ser cuidadosamente considerados (CHAMBERS \& NELSON, 1993; WAITES et al., 1988).

Os resultados desta pesquisa poderão úteis para o desenvolvimento de sistemas assépticos, bem como para outras aplicações no setor de industrialização de alimentos visando a redução da carga microbiana de matéria-prima, produtos e materiais de embalagem. Portanto, o objetivo deste estudo foi avaliar a esterilização de filme plástico de polietileno de baixa densidade, utilizando um protótipo equipado com lâmpadas germicidas.

\section{MATERIALE MÉTODOS}

Inicialmente, foram testadas 10 amostras de filme de polietileno de baixa densidade, usualmente empregados por indústrias de alimentos, a fim de verificar se eles apresentavam alguma contaminação inicial ao serem expostos ao ar ambiente. Para testar a radiação ultravioleta, o filme foi contaminado inicialmente por uma suspensão de esporos de Bacillus subtilis (ABREU \& FARIA, 2004).

Após esses ensaios, testaram-se várias intensidades e tempos diferentes a fim de obter o melhor planejamento experimental e a carga do inóculo inicial a ser utilizado.

Finalmente, com as variáveis independentes do planejamento experimental definida, foram realizados os ensaios definitivos a fim de comprovar a eficiência do método.

\section{Planejamento fatorial}

Objetivando-se avaliar a eficiência da radiação UV, foi utilizada uma seqüência de experimentos, determinada segundo metodologia de superfície de resposta (BOX et al., 1978). Executou-se um planejamento fatorial completo $2^{2}$ com 2 níveis $(-1,+1), 3$ pontos centrais (0) e 4 pontos axiais $(-\mathrm{a},+\mathrm{a})$, totalizando 11 ensaios. O planejamento considerou duas variáveis independentes: tempo de contato (s), distância da fonte $(\mathrm{cm})$, como observa-se na Figura 1. A resposta mensurável (variável dependente), foi a contagem de unidades formadoras de colônia de esporos sobreviventes (UFC)/ $\mathrm{cm}^{2}$, expressa em termos de reduções decimais $(\mathrm{g})$ :

$$
\gamma=\log \mathrm{N}_{0} / \mathrm{N}, \text { onde: }
$$

$\gamma=$ Número de reduções decimais atingido pelo tratamento; $\mathrm{N}_{0}=$ Número inicial (UFC/ $\mathrm{cm}^{2}$ da embalagem);

$\mathrm{N}=$ Número de sobreviventes (UFC/ $\mathrm{cm}^{2}$ da embalagem). 
Foram feitos testes preliminares com o objetivo de padronização da metodologia e definição dos pontos de análises, a fim de obter-se o melhor planejamento experimental e a carga ideal do inóculo inicial. Através desses testes preliminares, foram determinados os valores da variável independente e a carga do inóculo inicial que foi utilizado nos ensaios cujo valor foi aproximadamente $1,5 \times 10^{5}$ $\mathrm{UFC} / \mathrm{mL}$. O delineamento e a matriz do planejamento fatorial completo estão indicados nas Tabelas 1 e 2 , respectivamente.

\section{Preparo da suspensão de esporos}

A contagem do número de esporos de Bacillus subtilis foi feita em meio Agar Triptona Glicose Extrato de Carne (TGE), preparando-se uma suspensão até a carga inicial de $10^{9} \mathrm{UFC} / \mathrm{mL}$.

\section{Inoculação dos esporos}

Do filme plástico na forma de bobina, obtido da Dixie Toga Ltda, São Paulo-SP, foram retiradas amostras de 20 ' 15 $\mathrm{cm}$. Para aplicação dos esporos sob a superfície do filme, utilizou-se o procedimento descrito por Sizer et al. (1998) cujo método de contaminação foi denominado de "swab out".

Foram confeccionados moldes de aço inox com polimentos sanitários e esterilizáveis em autoclave (Figura 2), para delimitar a área de aplicação dos esporos e para a contagem.

\section{Quantificação dos esporos}

Amostras de $510 \mathrm{~cm}$ foram retiradas do filme e avaliadas quanto a carga inicial e para os testes de inoculação e posterior exposição à radiação UV. Após semear as placas, essas foram incubadas a $35^{\circ} \mathrm{C}$ por $48 \mathrm{~h}$. Através do procedimento descrito, determinou-se a carga microbiológica inicial em $50 \mathrm{~cm}^{2}$.

\section{Irradiação das amostras}

Para a irradiação dos filmes com ultravioleta, foi confeccionada uma câmara contendo lâmpadas emissoras de irradiação UV (254 nm), marca Light Electronics, referência LE F30T10/GL. O aparelho desenvolvido para a irradiação das embalagens, contendo a câmara, as lâmpadas germicidas e a esteira, está ilustrado pela Figura 3.

O filme plástico foi posicionado sobre a esteira de velocidade variável. A velocidade e a distância do filme das lâmpadas foram de acordo com o planejamento experimental descrito anteriormente.

Imediatamente após a irradiação, as amostras, obtidas com o auxílio do molde de aço inox de $5 \times 10 \mathrm{~cm}$, foram submetidas à contagem da carga microbiana. $\mathrm{O}$ procedimento para essa contagem foi o mesmo empregado na quantificação inicial de esporos na superfície delimitada pelo molde $\left(50 \mathrm{~cm}^{2}\right)$.

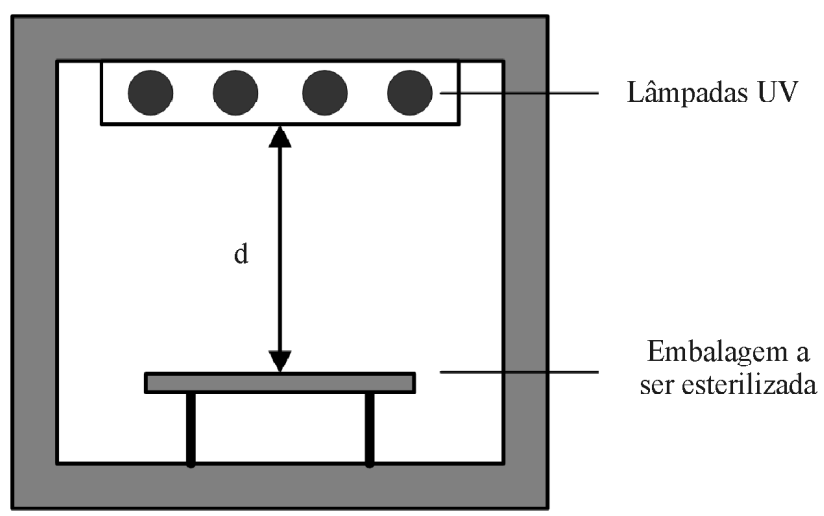

Figura 1 - Ilustração interna da câmara de irradiação ultravioleta.

Tabela 1 - Delineamento do planejamento fatorial completo $2^{2}$.

\begin{tabular}{cccccr}
\hline Níveis & $-1,44$ & -1 & 0 & 1 & 1,44 \\
\hline Tempo $(\mathrm{s})$ & 2,95 & 5 & 10 & 15 & 17,05 \\
$\mathrm{~d}(\mathrm{~cm})$ & 21,00 & 19,30 & 15,15 & 11,00 & 9,30 \\
\hline
\end{tabular}


Tabela 2 - Matriz do planejamento fatorial completo $2^{2}$.

\begin{tabular}{ccc}
\hline Ensaio & Tempo $(\mathrm{s})$ & $\mathrm{d}(\mathrm{cm})$ \\
\hline 1 & -1 & -1 \\
2 & -1 & +1 \\
3 & +1 & -1 \\
4 & +1 & +1 \\
5 & 0 & 0 \\
6 & 0 & 0 \\
7 & 0 & 0 \\
8 & $-1,44$ & 0 \\
9 & $+1,44$ & 0 \\
10 & 0 & $-1,44$ \\
11 & 0 & $+1,44$ \\
\hline
\end{tabular}
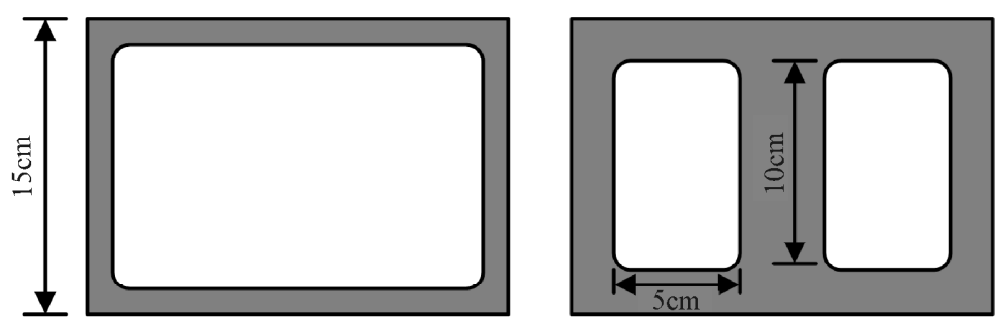

Figura 2 - Moldes utilizados na contagem inicial e final de esporos.

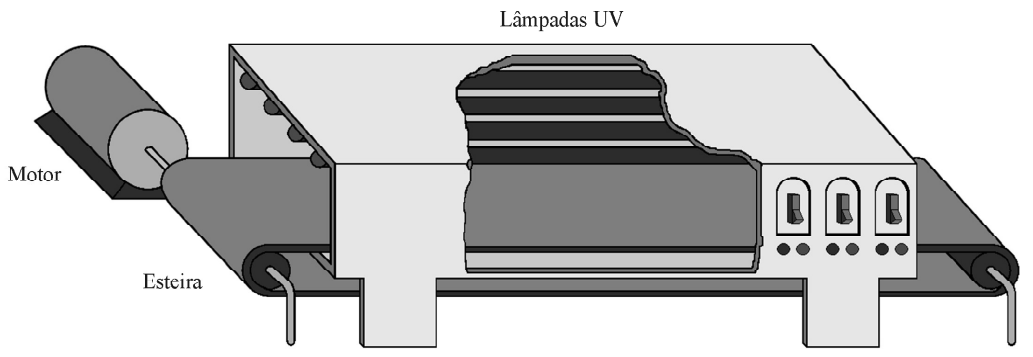

Figura 3 - Aparelho desenvolvido para a irradiação das embalagens.

\section{RESULTADOS E DISCUSSÃO}

Os resultados dos ensaios, com base no planejamento experimental, encontram-se transcritos na Tabela 3. Nessa tabela, verifica-se que a redução decimal variou de 0,21 a 2,47, dependendo do binômio tempo e distância utilizados.

Stannnard et al. (1983) verificaram que, para o tempo de $10 \mathrm{~s}$ e a distância de $4 \mathrm{~cm}$ da fonte, o número de reduções decimais dos esporos de Bacillus subtilis foi de 1,9, inoculado inicialmente com $10^{7}$ UFC. Stannard et al. (1985) também verificaram redução decimal de 2,63 em embalagens de polietileno expostas a $10 \mathrm{~cm}$ da lâmpada germicida, utilizando esporo de Bacillus subtilis como microrganismo teste.
Bayliss \& Waites (1979) demonstraram que, para o tempo de $30 \mathrm{~s}$ e a distância da fonte de $5,5 \mathrm{~cm}$, a porcentagem de sobreviventes de Bacillus subtilis foi de 1,44.

Segundo a Tabela 4, obtida através do programa STATISTICA 5.0, o valor do efeito linear do tempo foi positivo, enquanto o valor do efeito linear da distância foi negativo. Isso comprova o fato de que, quanto maior o tempo de exposição à radiação UV, maior a redução decimal e quanto maior a distância da embalagem da fonte UV, menor a redução decimal. Já os valores quadráticos, tanto da distância quanto do tempo são negativos, ou seja, ambos quando aumentados provocam menor redução decimal. 
Tabela 3 - Resultados das reduções decimais causadas pela irradiação UV.

\begin{tabular}{cccc}
\hline Ensaio & Tempo $(\mathrm{s})$ & $\mathrm{d}(\mathrm{cm})$ & $\gamma$ \\
\hline 1 & -1 & -1 & 0,21 \\
2 & -1 & 1 & 0,63 \\
3 & 1 & -1 & 2,32 \\
4 & 1 & 1 & 2,47 \\
5 & 0 & 0 & 2,02 \\
6 & 0 & 0 & 2,05 \\
7 & 0 & 0 & 1,99 \\
8 & $-1,44$ & 0 & 0,77 \\
9 & 1,44 & 0 & 2,10 \\
10 & 0 & $-1,44$ & 0,60 \\
11 & 0 & 1,44 & 2,20 \\
\hline
\end{tabular}

Tabela 4 - Cálculo do efeito e dos coeficientes referentes aos tratamentos.

\begin{tabular}{lcccc}
\hline & Efeito & Efeito percentual & Efeito absoluto & Coeficientes \\
\hline Principal interação & 2,02 & 116,6276 & 0,000074 & 2,02 \\
Tempo Linear & 1,46 & 68,7542 & 0,000211 & 0,73 \\
Tempo Quadrático & $-0,59$ & $-23,3424$ & 0,001830 & $-0,30$ \\
Distância Linear & $-0,71$ & $-33,3544$ & 0,000898 & $-0,35$ \\
Distância Quadrático & $-0,63$ & $-24,7313$ & 0,001631 & $-0,31$ \\
\hline
\end{tabular}

Na Tabela 4 também quantificam-se os valores do efeito da irradiação de forma absoluta e quantificam-se porcentual. Através desses resultados, verifica-se que tanto o tempo quanto a distância tem grande influência nos resultados, apresentando efeito linear e quadrático.

A partir dos dados da Tabela 4, calcularam-se os valores dos coeficientes da curva cuja equação foi:

$$
Y=2,02+0,730 \times t-0,30 \times t^{2}-0,35 d-0,31 \times d^{2}+0,068 \times t \times d
$$

Onde:

$\mathrm{Y}=$ número de reduções decimais;

$t=$ tempo de exposição da embalagem à radiação UV (s); $d=$ distância da embalagem à fonte $(\mathrm{cm})$.

A Tabela 5 ilustra os resultados obtidos dos testes preliminares. Nos experimentos 2, 3, 4 e 5 houve uma redução total de microrganismos. Por esse motivo não foi possível determinar de forma precisa o número de reduções decimais já que, nesse tipo de metodologia, o valor que realmente importava era a diferença entre a contagem de microrganismos finais e iniciais.
A partir dos dados da Tabela 4 foi possível também a visualização global do efeito da irradiação e das variáveis, ao construir-se a superfície de resposta e curvas de contorno dos resultados experimentais (Figuras 4 e 5). Observou-se que, quanto menor a distância da fonte e maior o tempo de exposição à irradiação UV, maior foi o número de reduções decimais.

A dose normalmente aplicada na esterilização está em torno de $10^{5} \mathrm{~mW} . \mathrm{s} / \mathrm{cm}^{2}$, valor esses tão baixos, que não chegam a provocar aumento de temperatura da amostra irradiada. Sabe-se que o efeito potencialmente de maior letalidade ocorre no ácido desoxirribonucléico da célula, principalmente em conseqüência da formação de dímeros de nucleotídeos que são os mais abundantes e estáveis produtos resultantes da irradiação. As irradiações inibem a síntese de ácido desoxirribonucléico e, em menor extensão, a de ácido ribonucléico e a síntese de proteínas (INGRAM \& ROBERTS, 1980).

Cardoso (2007) obteve 1 redução decimal na carga inicial em esporos de Bacillus subtilis, inoculados em embalagens de polietileno de baixa densidade, após exposição à radiação UV. Quando o mesmo tratamento foi repetido, porém com prévia imersão em solução de peróxido de hidrogênio (18\%), atingiu-se 7 reduções decimais, demonstrando nesse caso o efeito sinérgico da radiação UV. 
Tabela 5 - Efeito do tempo de exposição sobre as reduções decimais de Bacillus subtilis.

\begin{tabular}{cccc}
\hline Experimentos & Tempo & $\mathrm{d}(\mathrm{cm})$ & Reduções decimais \\
\hline 1 & 15 & 21,00 & 2,40 \\
2 & 30 & 21,00 & 5,33 \\
3 & 20 & 21,00 & 4,00 \\
4 & 15 & 9,50 & 2,00 \\
5 & 15 & 10,50 & 4,62 \\
\hline
\end{tabular}
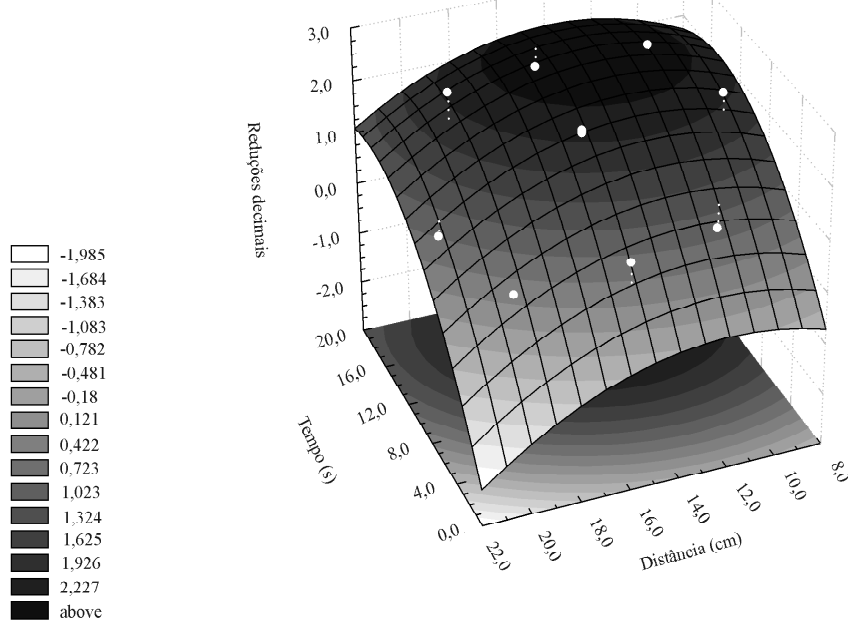

Figura 4 - Superfície de resposta das reduções decimais de Bacillus subtilis, sob efeito da fonte de UV.

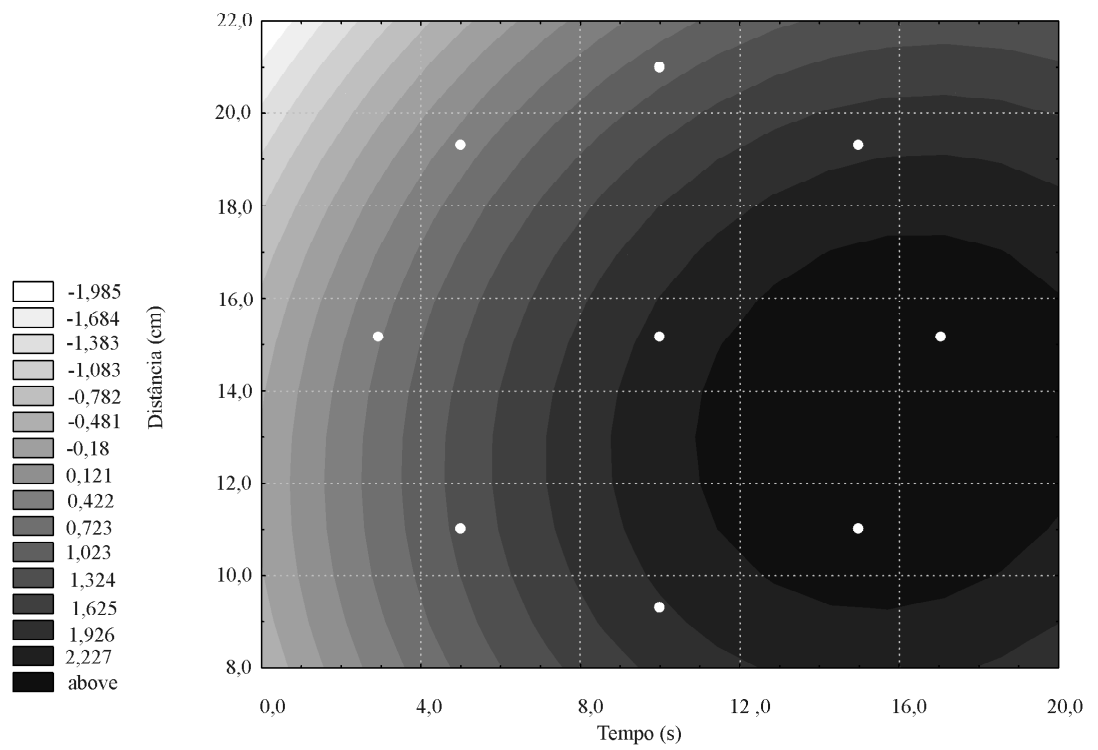

Figura 5 - Curvas de contorno das reduções decimais em função do tempo de exposição e distância da fonte. 


\section{CONCLUSÃO}

As reduções decimais dos esporos de Bacillus subtilis inoculados sobre a superfície de filmes variaram de 0,21 a 2,47, dependendo do binômio tempo e distância utilizados. Quanto maior o tempo de exposição, maior é o efeito e, neste experimento, quando o tempo foi superior a 30 s houve uma redução total da carga microbiana. Portanto, através do trabalho foi possível concluir que esse sistema de esterilização de superfície de embalagens é eficaz, principalmente quando o tempo de exposição do material à irradiação UV $254 \mathrm{~nm}$ for superior a $30 \mathrm{~s}$. Concluiu-se, também, que a distância da superfície a ser irradiada deve ser a mínima possível para aumentar o efeito germicida das fontes de ultravioleta.

\section{REFERÊNCIAS BIBLIOGRÁFICAS}

ABREU, L. F.; FARIA, J. A. F. Evaluation of a system for chemical sterilization of packages. Packaging Technology and Science, v. 17, p. 37-42, 2004.

BAYLISS, C. E.; WAITES, W. M. The combined effect of hydrogen peroxide and ultraviolet irradiation on bacterial spores. Journal of Applied Bacteriology, v. 47, p. 236-269, 1979.

BOX, G. E. P.; HUNTER, W. G.; HUNTER, J. S. Statistics for experiments: an introduction to design, data analyses and model building. Nova York: J. Wiley \& Sons, 1978. 645 p.

CARDOSO, C. F. Avaliação da esterilização de filme de polietileno com peróxido de hidrogênio e radiação ultravioleta. 2007. Dissertação (Mestrado em Tecnologia de Alimentos) - Universidade Estadual de Campinas, Campinas, 2007.

CHAMBERS, J. V.; NELSON, P. E. Principles of aseptic processing and packaging. Washington, DC: The Food Processors Institute, 1993. 257 p.

FARIA, J. A. F. Sistema de esterilização de embalagens. BR Instituto Nacional de Propriedades Industrial (INPI). Patente n. 1427, 2001.

FARIA, J. A. F. Vida de prateleira de alimentos processados assepticamente. In: SEMINÁRIO LATINO AMERICANO - AVANÇOS EM TECNOLOGIA PARA O PROCESSAMENTO E ENVASAMENTO ASSÉPTICO DE ALIMENTOS, 2., 1993, Campinas, SP. Anais... Campinas: Unicamp, 1993. p. 7-28.
INGRAM, M.; ROBERTS, T. A. Application of the " $D$ concept" to heat treatments involving curing salts. Journal Food Technology, v. 6, p. 21-28, 1980.

INTERNATIONAL COMISSION ON MICROBIOLOGICAL SPECIFICATION FOR FOODS. Microbial ecology of foods. New York: Academic, 1980. v. 1.

JOYCE, D. A. microbiological aspects of aseptic processing and packaging. In: WILHOFT, E. M. A. Aseptic processing and packaging of particulate foods. Glasgow: Blackie Academic \& Professional, 1993. chap. 8, p. 155-180.

KONINKLIJIKEPHILIPS ELETRONICS. UV disinfection: application information. Disponível em: <http:// IWwW.philips.com/lighting >. Acesso em: 20 abr. 2007.

REUTER, H. Processes for packaging material sterilization and system requirements. In: of foods. Hamburg: Technomic, 1993 Aseptic processing

SIEMENS WATER TECHNOLOGIES. UV technology: chemical-free water treatment and disinfection. Disponível em: 〈http://www.siemens.com >. Acesso em: 27 abr. 2007.

SIZER, C.; PALANIAPPAN, S.; HOLBERT, V. Method and apparatus for sterilizing packaging. A61L 002/00. US Patent 5843374. 9218170 WO. 10 jan. 1992. Tetra Laval Holdings \& Finance Pully/CH, n. 527, p. 15, 1 dez. 1998.

STANNARD, C. J.; ABISS, J. S.; WOOD, J. M. Combined treatment with hydrogen peroxide and ultra-violet irradiation to reduce microbial contamination levels in preformed food packaging cartons. Journal of Food Protection, v. 46, n. 12, p. 1060-1064, 1983.

STANNARD, C. J.; ABISS, J. S.; WOOD, J. M. Efficiency of treatments involving ultraviolet irradiation for decontaminating packaging board of different surface compositions. Journal of Food Protection, v. 48, n. 9, p. 786-789, 1985.

WAITES, W. M.; HARDING, S. E.; FOWLER, D. R.; JONES, S. H.; SHAW, D.; MARTIN, M. The destruction of spores of Bacillus subtilis by the combined effects of hydrogen peroxide and ultraviolet light. Letters in Applied Microbiology, v. 7, p. 139-140, 1988.

WILLHOFT, E. M. A. Aseptic processing and packaging of particulate foods. Glasgow: Blackie Academic \& Professional, 1993. chap. 2, p. 23-38. 